\title{
Meconium Indicators of Maternal Alcohol Abuse during Pregnancy and Association with Patient Characteristics
}

\author{
Tamme W. Goecke, ${ }^{1,2}$ Pascal Burger, ${ }^{3}$ Peter A. Fasching, ${ }^{1}$ \\ Abdulsallam Bakdash, ${ }^{4}$ Anne Engel, ${ }^{1}$ Lothar Häberle, ${ }^{1}$ Franziska Voigt, ${ }^{1,2}$ \\ Florian Faschingbauer, ${ }^{1}$ Eva Raabe, ${ }^{1}$ Nicolai Maass, ${ }^{2}$ Michael Rothe, ${ }^{5}$ \\ Matthias W. Beckmann, ${ }^{1}$ Fritz Pragst, ${ }^{4}$ and Johannes Kornhuber ${ }^{3}$ \\ ${ }^{1}$ Department of Obstetrics and Gynecology, Friedrich-Alexander-University of Erlangen-Nuremberg, Universitaetsstraß 21-23, \\ 91054 Erlangen, Germany \\ ${ }^{2}$ Department of Obstetrics and Gynecology, University of RWTH Aachen, Pauwelsstrß 30, 52074 Aachen, Germany \\ ${ }^{3}$ Department of Psychiatry and Psychotherapy, Friedrich-Alexander-University of Erlangen-Nuremberg, Schwabachanlage 6-10, \\ 91054 Erlangen, Germany \\ ${ }^{4}$ Institute of Legal Medicine, University Hospital Charité, Hittorfstraße 18, 14195 Berlin, Germany \\ ${ }^{5}$ Lipidomix GmbH, Berliner Allee 261-269, 13088 Berlin, Germany
}

Correspondence should be addressed to Tamme W. Goecke; tgoecke@ukaachen.de

Received 12 January 2014; Revised 12 February 2014; Accepted 12 February 2014; Published 30 March 2014

Academic Editor: Gottfried E. Konecny

Copyright ( 2014 Tamme W. Goecke et al. This is an open access article distributed under the Creative Commons Attribution License, which permits unrestricted use, distribution, and reproduction in any medium, provided the original work is properly cited.

\begin{abstract}
Aim. Identification of women with moderate alcohol abuse during pregnancy is difficult. We correlated self-reported alcohol consumption during pregnancy and patient characteristics with objective alcohol indicators measured in fetal meconium. Methods. A total of 557 women singleton births and available psychological tests, obstetric data and meconium samples were included in statistical analysis. Alcohol metabolites (fatty acid ethyl esters (FAEEs) and ethyl glucuronide (EtG)), were determined from meconium and correlated with patient characteristics. Results. We found that $21.2 \%$ of the 557 participants admitted low-tomoderate alcohol consumption during pregnancy. Of the parameters analyzed from meconium, only EtG showed an association with alcohol history $(P<0.01)$. This association was inverse in cases with EtG value above $120 \mathrm{ng} / \mathrm{g}$. These values indicate women with most severe alcohol consumption, who obviously denied having consumed alcohol during pregnancy. No other associations between socioeconomic or psychological characteristics and the drinking status (via meconium alcohol metabolites) could be found. Conclusion. Women who drink higher doses of ethanol during pregnancy, according to metabolite measures in meconium, might be less likely to admit alcohol consumption. No profile of socioeconomic or psychological characteristics of those women positively tested via meconium could be established.
\end{abstract}

\section{Introduction}

Self-reported maternal alcohol abuse during pregnancy is not reliable and ethanol consumption is rarely admitted, if at all $[1,2]$. However, alcohol consumption during pregnancy is a relevant problem, with its estimated prevalence ranging from 3.5 up to 53.9\% in European countries [1-3]. Excessive prenatal alcohol exposure is reported to be associated with severe consequences for the fetus, such as premature birth, miscarriages, fetal alcohol syndrome (FAS) or fetal alcohol spectrum disease (FASD), and other physical and neuropsychological disorders [4-6].

Even low or moderate alcohol exposure may lead to a higher perinatal mortality [7], and it may cause congenital anomalies. In addition, the risk increases with higher dose $[8,9]$. The role of moderate alcohol consumption in pregnant women is controversially discussed $[3,10-15]$, particularly because an exact dose-effect correlation between alcohol 
intake and development of physical and neuropsychological problems could not be established. Therefore, a labeling for alcoholic drinks and a recommendation of complete abstinence during pregnancy have been established in many European countries, such as France.

Commonly performed laboratory tests for alcohol consumption such as carbohydrate deficient transferrin (CDT), liver enzymes such as gamma-glutamyl-transferase (GGT), and mean corpuscular volume (MCV) are indirect alcohol markers. They are difficult to interpret and insufficiently reliable during pregnancy $[16,17]$.

Other parameters for alcohol consumption exist as direct metabolites of ethanol degradation. They can be found in blood, urine, hair, and meconium. Fatty acid ethyl esters (FAEE) in meconium have been investigated and established in several studies as biomarkers of fetal ethanol exposure during the last 3 months of pregnancy $[1,18-20]$. Additionally, the determination of ethylglucuronide $(\mathrm{EtG})$ not only from the mothers' hair or urine but also from the children's meconium has been associated with the mother's drinking behavior during pregnancy [2, 20-23].

In our study, we aimed at the assessment of the association between patients' self-reported alcohol intake and meconium biomarkers for maternal alcohol consumption.

Furthermore, we tried to identify the characteristics of mothers, who, according to direct ethanol metabolites, presumably drank during their pregnancy, using epidemiological and medical history, and we standardized the psychological questionnaires.

\section{Materials and Methods}

The Franconian Maternal Health Evaluation Studies (FRAMES) were prospectively conducted from 2005 to 2007 [24-27]. A total of 1100 women were recruited as a consecutive cohort. The participating women had to be aged $\geq 18$ years with at least 30 full weeks of gestational age, and were introduced as outpatients to the Department of Obstetrics and Gynecology of the University of ErlangenNuremberg. There was no preselection of the cases with respect to suspected alcohol abuse of the mother or any other parameter.

Only singleton births were allowed for this analysis, resulting in the exclusion of 114 births. Therefore, the final number of participants in this study was 986. Further, 247 newborns were transferred to the children's hospital because of perinatal problems and were excluded as well. Of the left 739 newborns, 137 had to be excluded because of missing consent of the mother or sampling was missed. From the remaining 602 samples, 45 could not be investigated due to technical issues (i.e., too little sample volume). There were no statistical differences between women with available meconium measurements (557) and those without.

This study was approved by the Local Ethics Committee of the Medical Faculty of the University of ErlangenNuremberg and was conducted in accordance with the Declaration of Helsinki and all patients gave informed consent.
All participating women were interviewed with standardized psychological questionnaires for the identification of comorbid psychiatric disorders at three occasions. The first was done prenatally from the 30th week of pregnancy onward (first contact with the pregnant women), the second was done 48-72 hours postpartum (when the meconium was collected), and the third was carried out 6-8 months postpartum. Furthermore, we collected diagnostic, obstetric, and medical history from the women.

Psychological questionnaires for other psychiatric disorders were comprised, including the Hamilton rating scale for depression (HAMD), the Edinburgh Postnatal Depression Scale (EPDS), and others - the results of these evaluations are published elsewhere. In addition, anamnestic data on partnership, sexual life, and social status as well as medical parameters of the mother and child were acquired.

About $1 \mathrm{~g}$ of meconium was collected from the newborns within the first $2-24 \mathrm{~h}$ after birth and frozen at $-80^{\circ} \mathrm{C}$ for up to 30 months until analysis. The meconium samples were analyzed in the Department of Forensic Toxicology of the Institute of Legal Medicine, University Hospital Charité, and by the Lipidomix $\mathrm{GmbH}$ in Berlin. The procedure for determination of FAEE and EtG has been described in detail in a previous paper [20]. The FAEEs ethyl Myristate (E14), ethyl Palmitate (E16), ethyl Linoleate (E18:2), ethyl Oleate (E18:1), and ethyl Stearate (E18), as well as the corresponding deuterated standards D5-FAEE, were purchased or prepared as described previously $[20,28]$. The quantification of FAEE in meconium was performed according to an optimized and validated method described previously [23].

EtG was determined in meconium according to a liquidchromatography/tandem-mass-spectrometry (LC-MS-MS) procedure with D5-EtG as the internal standard in analogy to the measurement in hair [21]. The measurement was performed by LC-MS-MS as described in our previous paper [20].

2.1. Statistical Considerations. Univariant associations of alcohol history, meconium results, and socioeconomic parameters were analyzed with appropriate statistical tests. Wilcoxon rank-sum tests were used for ordinal parameters, and $\chi^{2}$ tests were used for categorical (i.e., yes/no) parameters. All measures of meconium concentrations are reported in ng per $\mathrm{g}$ meconium.

In a preanalysis, boxplots were generated to get a first impression of the meconium data distributions in the two groups of reported alcohol consumption: yes (Y) and no $(\mathrm{N})$. The distributions were asymmetric with many outliers in the group with no reported alcohol consumption as well as higher values for the majority of measurements in the group with reported alcohol consumption (Figure 1). We therefore hypothesized a cutoff point, which could divide the women into two groups: one with at least moderate measurements, which would have a positive association of the meconium results with alcohol history, and a second with higher meconium results, in which this association would be negative. To find an optimal cutoff, thresholds were run between the 10th and the 90th percentile of all 

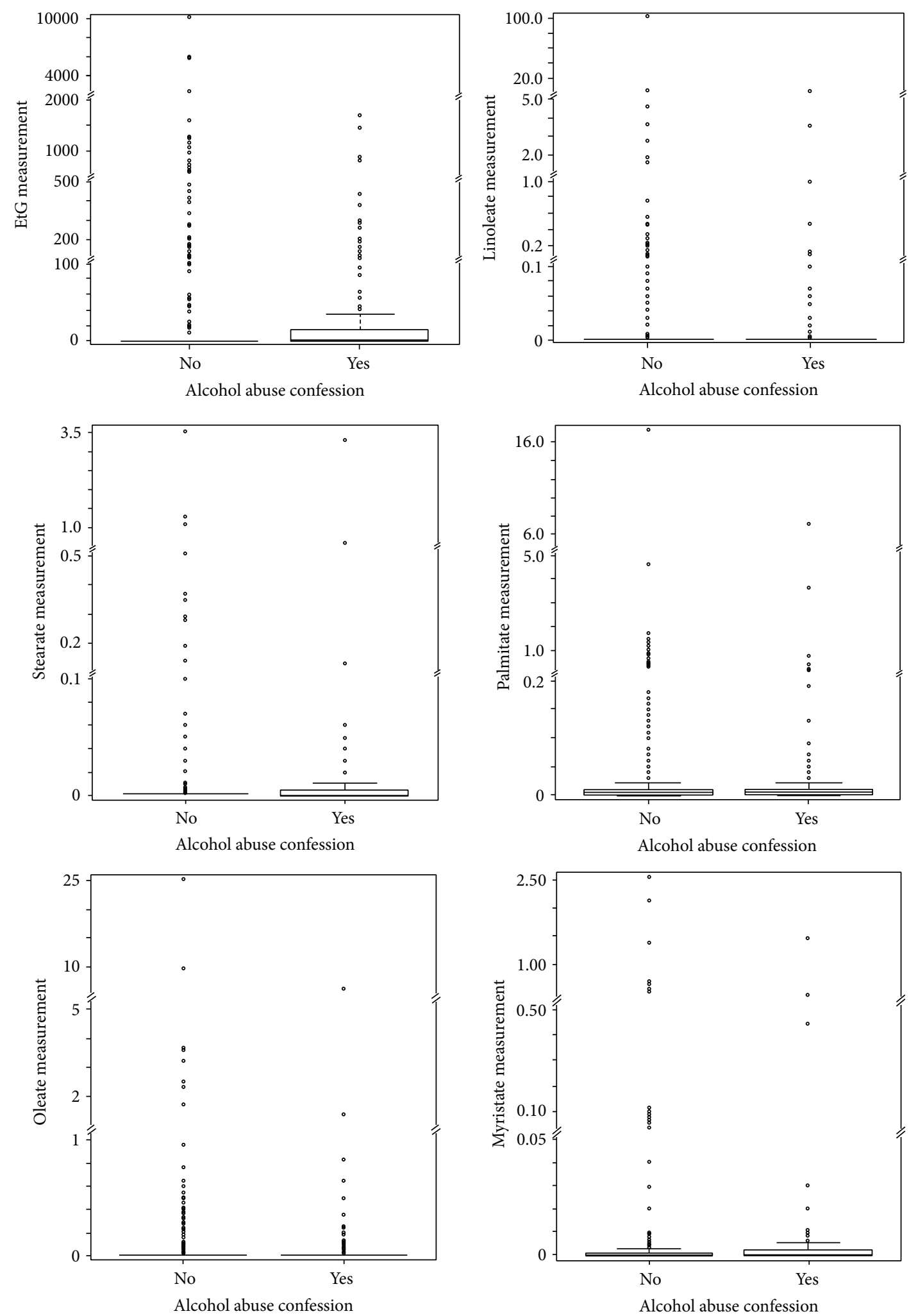

Figure 1: Boxplots of EtG and ester measures and alcohol abuse confession. Suitable ranges are displayed. 
TABLE 1: Association of meconium results, report of alcohol consumption count and percentage, and $P$ values of Wilcoxon rank-sum tests are shown (test based on raw values, classifications just for illustration).

\begin{tabular}{|c|c|c|c|c|c|}
\hline Meconium results & $\mathrm{ng} / \mathrm{g}$ & Total & No alcohol abuse reported & Alcohol abuse reported & $P$ value \\
\hline \multirow{2}{*}{ Stearate } & 0 & 411 & $80.8 \%$ & $19.2 \%$ & \multirow{2}{*}{0.08} \\
\hline & $>0$ & 139 & $72.7 \%$ & $27.3 \%$ & \\
\hline \multirow{2}{*}{ Linoleate } & 0 & 460 & $79.1 \%$ & $20.9 \%$ & \multirow{2}{*}{0.66} \\
\hline & $>0$ & 90 & $76.7 \%$ & $23.3 \%$ & \\
\hline \multirow{2}{*}{ Oleate } & 0 & 416 & $78.8 \%$ & $21.2 \%$ & \multirow{2}{*}{0.75} \\
\hline & $>0$ & 134 & $78.4 \%$ & $21.6 \%$ & \\
\hline \multirow{2}{*}{ Palmitate } & 0 & 121 & $80.2 \%$ & $19.8 \%$ & \multirow{2}{*}{0.55} \\
\hline & $>0$ & 423 & $78.5 \%$ & $21.5 \%$ & \\
\hline \multirow{2}{*}{ Myristate } & 0 & 367 & $79.3 \%$ & $20.7 \%$ & \multirow{2}{*}{0.76} \\
\hline & $>0$ & 183 & $77.6 \%$ & $22.4 \%$ & \\
\hline \multirow{2}{*}{ EtG } & 0 & 451 & $81.4 \%$ & $18.6 \%$ & \multirow{2}{*}{$<0.01$} \\
\hline & $>0$ & 92 & $66.3 \%$ & $33.7 \%$ & \\
\hline
\end{tabular}

meconium measurements. For each choice of the threshold, both the subgroups (Group $1<$ cutoff, Group $2 \geq$ cutoff) were separately tested to determine the differences between the abusers and nonabusers with Wilcoxon rank-sum tests. The optimal cutoff point was defined as the minimum sum of $P$ values from both tests.

Multiple logistic regression models with meconium measures ( $=0$ versus $>0$, resp., <cutoff point versus $\geq$ cutoff point) as target variables and socioeconomic parameters as predictive variables were performed to calculate the odds ratios (OR). The final models were obtained by the backward stepwise variable selection due to the Akaike information criterion. For each model, the area under the curve (AUC) of the receiver operation characteristic (ROC), ranging from 0.5 (random prediction) to 1 (perfect prediction), was calculated to summarize the strength of prediction.

All tests were two-sided, and a $P$ value of $<0.05$ was considered to be statistically significant. The analyses were carried out using the $\mathrm{R}$ system for statistical computing (version 2.11.1; R Development Core Team, Vienna, Austria, 2010).

\section{Results}

3.1. Main Patient Characteristics. The final number of participating women with singleton birth in this study was 986 . In average, participants were 32 years old and 557 gave birth to their first child in this study. Average pregnancy at the time of birth was 1.9; 514 were boys and 470 were girls. The average weight for the boys was $3497 \mathrm{~g}$ and the average weight for the girls was $3318 \mathrm{~g}$.

A total of 204 women (20.8\%) confessed to have drunk alcohol at some time point to some extent during pregnancy. Most women stated to have drunk moderately alcohol with a low frequency. None of the women admitted to having drunk alcohol extensively.

3.2. Main Measurement Characteristics. With regard to the meconium measures (Linoleate, Palmitate, Stearate, Oleate, Myristate, and EtG), most of the samples were free of fatty esters and EtG. The percentage of negative samples reached from $22 \%$ to $84 \%$ in the group of the fatty esters and was $83 \%$ for EtG. The maximum values were rather low for Myristate and Stearate with 2.6 and 3.6, respectively, and reached values of 103.2 with regard to Linoleate. Oleate and Palmitate maximum values were in between with 25.4 and 17.4. Maximum value for EtG was 10,235. The distribution of measurements is shown in Figure 1.

3.3. Association of Alcohol Abuse Confession with Meconium Measures. For the majority of meconium measures, the strong outliers seen in the boxplots of the meconium measures result in a higher mean in the group of alcohol abuse confessing women compared to the group of women who did not confess alcohol abuse. The mean EtG value in the group of alcohol abuse confessing women was 71 and it was 110 in the group of women who did not confess alcohol abuse. The corresponding values for Linoleate were 0.30 versus 0.05 , for Oleate were 0.16 versus 0.11 , and for Myristate were 0.023 versus 0.018 .

Rank based testing confirmed only for EtG the observation that the group with alcohol abuse confession showed overall higher measurements $(P<0.01$, Wilcoxon ranksum test) (Table 1). Additionally, the five meconium measures Linoleate, Palmitate, Stearate, Oleate, and Myristate were analyzed with other statistical methods without any significant results (data not shown).

Only for EtG, an optimal cutoff with significant $P$ values was found. In fact, this cutoff was at $120 \mathrm{ng} / \mathrm{g}$ for EtG. When looking only at the group of women with an EtG $<120 \mathrm{ng} / \mathrm{g}$, those women denying alcohol abuse had mean EtG values of $3.0 \mathrm{ng} / \mathrm{mL}$, while those women admitting alcohol abuse had mean EtG values of $6.6 \mathrm{ng} / \mathrm{mL}(P<0.001)$. Above the cutoff, the mean EtG value was 482.2 for women with alcohol abuse confession and 1179.8 for women without alcohol abuse confession $(P=0.055)$. For all other meconium measures, the optimal cut point had no significant $P$ values (data not shown). Due to small sample size, this cutoff could not be validated. 
TABLE 2: Association of socioeconomic parameters and EtG measures. Count, percentage, and $P$ value of nonparametric test are shown.

\begin{tabular}{|c|c|c|c|c|}
\hline Patient characteristic & Total & EtG measurement $<120 \mathrm{ng} / \mathrm{g}$ & EtG measurement $\geq 120 \mathrm{ng} / \mathrm{g}$ & $P$ value \\
\hline \multicolumn{5}{|l|}{ Age } \\
\hline$<20$ & 3 & $66.7 \%$ & $33.3 \%$ & \\
\hline $20-30$ & 128 & $90.6 \%$ & $9.4 \%$ & \\
\hline $30-40$ & 382 & $90.1 \%$ & $9.9 \%$ & \\
\hline $40+$ & 32 & $90.6 \%$ & $9.4 \%$ & \\
\hline Total & 545 & & & 0.92 \\
\hline \multicolumn{5}{|l|}{ Education } \\
\hline No university-entrance diploma & 245 & $91.8 \%$ & $8.2 \%$ & \\
\hline University-entrance diploma & 296 & $88.5 \%$ & $11.5 \%$ & \\
\hline Total & 541 & & & 0.25 \\
\hline \multicolumn{5}{|l|}{ Marital status } \\
\hline Not married & 110 & $84.5 \%$ & $15.5 \%$ & \\
\hline Married & 434 & $91.5 \%$ & $8.5 \%$ & \\
\hline Total & 544 & & & 0.05 \\
\hline \multicolumn{5}{|l|}{ Income } \\
\hline$<500 €$ & 1 & $0.0 \%$ & $0.0 \%$ & \\
\hline $500-1000 €$ & 19 & $100.0 \%$ & $0.0 \%$ & \\
\hline $1000-2000 €$ & 59 & $89.8 \%$ & $10.2 \%$ & \\
\hline $2000-3000 €$ & 122 & $85.2 \%$ & $14.8 \%$ & \\
\hline $3000-4000 €$ & 94 & $94.7 \%$ & $5.3 \%$ & \\
\hline $4000-5000 €$ & 56 & $89.3 \%$ & $10.7 \%$ & \\
\hline$>5000 €$ & 48 & $93.8 \%$ & $6.3 \%$ & \\
\hline Total & 399 & & & 0.32 \\
\hline \multicolumn{5}{|l|}{ Smoking } \\
\hline No & 503 & $90.1 \%$ & $9.9 \%$ & \\
\hline Yes & 40 & $90.0 \%$ & $10.0 \%$ & \\
\hline Total & 543 & & & 1.00 \\
\hline \multicolumn{5}{|l|}{ EPDS } \\
\hline No depression & 428 & $90.0 \%$ & $10.0 \%$ & \\
\hline Slight depression & 37 & $89.2 \%$ & $10.8 \%$ & \\
\hline Moderate-to-strong depression & 36 & $91.7 \%$ & $8.3 \%$ & \\
\hline Total & 501 & & & 0.88 \\
\hline \multicolumn{5}{|l|}{ HAMD } \\
\hline No depression & 466 & $91.8 \%$ & $8.2 \%$ & \\
\hline Slight-to-moderate depression & 56 & $91.1 \%$ & $8.9 \%$ & \\
\hline Strong depression & 11 & $90.9 \%$ & $9.1 \%$ & \\
\hline Total & 533 & & & 0.77 \\
\hline
\end{tabular}

Based on these results, further analyses with the alcohol abuse confession as target variable were conducted separately for the two subgroups defined by the EtG cutoff point.

3.4. Association of Meconium Measures with Socioeconomic Parameters. In order to understand whether there is a correlation between commonly known socioeconomic factors which might be correlated with alcohol abuse, both, the alcohol abuse confession variable and the meconium measures, were correlated with socioeconomic characteristics. Except for an association between smoking status and Oleate measures and marital status and Myristate measures, no associations were seen. Women who were smokers had more often an elevated Oleate measure (38.1\% versus $23.2 \%$; $P=$ 0.049 ), and women who were married had less frequently elevated Myristate measures (30.2\% versus 45.9\%; $P<$ $0.01)$. None of these tests were adjusted for multiple testing though, and none of the findings were consistent with other fatty ester measures or EtG. The associations between EtG and socioeconomic parameters for EtG with the determined cutoff of $120 \mathrm{ng} / \mathrm{g}$ are shown in Table 2.

3.5. Association between Alcohol Abuse Confession and Socioeconomic Factors. Comparing the women's statements about alcohol abuse confession revealed only an association 
TABLE 3: Association of socioeconomic parameters and alcohol for Group 1 (EtG < cutoff of $120 \mathrm{ng} / \mathrm{g}$ ). Count, percentage, and $P$ value of nonparametric test are shown.

\begin{tabular}{|c|c|c|c|c|}
\hline Patient characteristics & Total & Alcohol consumption not reported in Group 1 & Alcohol consumption reported in Group 1 & $P$ value \\
\hline \multicolumn{5}{|l|}{ Age } \\
\hline$<20$ & 2 & $100.0 \%$ & $0.0 \%$ & \\
\hline $20-30$ & 116 & $81.9 \%$ & $18.1 \%$ & \\
\hline $30-40$ & 342 & $78.4 \%$ & $21.6 \%$ & \\
\hline $40+$ & 29 & $82.8 \%$ & $17.2 \%$ & \\
\hline Total & 489 & & & 0.15 \\
\hline \multicolumn{5}{|l|}{ Education } \\
\hline No university-entrance diploma & a 223 & $78.0 \%$ & $22.0 \%$ & \\
\hline University-entrance diploma & 262 & $80.9 \%$ & $19.1 \%$ & \\
\hline Total & 485 & & & 0.50 \\
\hline \multicolumn{5}{|l|}{ Marital status } \\
\hline Not married & 93 & $78.5 \%$ & $21.5 \%$ & \\
\hline Married & 395 & $79.7 \%$ & $20.3 \%$ & \\
\hline Total & 488 & & & 0.90 \\
\hline \multicolumn{5}{|l|}{ Income } \\
\hline$<500 €$ & 0 & $0.0 \%$ & $0.0 \%$ & \\
\hline $500-1000 €$ & 19 & $84.2 \%$ & $15.8 \%$ & \\
\hline $1000-2000 €$ & 53 & $83.0 \%$ & $17.0 \%$ & \\
\hline $2000-3000 €$ & 104 & $83.7 \%$ & $16.3 \%$ & \\
\hline $3000-4000 €$ & 89 & $79.8 \%$ & $20.2 \%$ & \\
\hline $4000-5000 €$ & 50 & $78.0 \%$ & $22.0 \%$ & \\
\hline$>5000 €$ & 45 & $77.8 \%$ & $22.2 \%$ & \\
\hline Total & 360 & & & 0.27 \\
\hline \multicolumn{5}{|l|}{ Smoking } \\
\hline No & 453 & $79.0 \%$ & $21.0 \%$ & \\
\hline Yes & 36 & $86.1 \%$ & $13.9 \%$ & \\
\hline Total & 489 & & & 0.42 \\
\hline \multicolumn{5}{|l|}{ EPDS } \\
\hline No depression & 385 & $78.2 \%$ & $21.8 \%$ & \\
\hline Slight depression & 33 & $84.8 \%$ & $15.2 \%$ & \\
\hline Moderate-to-strong depression & 33 & $78.8 \%$ & $21.2 \%$ & \\
\hline Total & 451 & & & 0.54 \\
\hline \multicolumn{5}{|l|}{ HAMD } \\
\hline No depression & 428 & $79.2 \%$ & $20.8 \%$ & \\
\hline Slight-to-moderate depression & 51 & $80.4 \%$ & $19.6 \%$ & \\
\hline Strong depression & 10 & $90.0 \%$ & $10.0 \%$ & \\
\hline Total & 489 & & & 0.59 \\
\hline
\end{tabular}

between alcohol abuse and age of women with an EtG measure above $120 \mathrm{ng} / \mathrm{g}$ (Tables 3 and 4).

3.6. Multivariate Models. Thinking of objective alcohol meconium measures as variables which might be helpful in clinical practice to predict, socioeconomic factors and alcohol abuse confession as independent variables, and meconium measures as dependent variables (six unique models) were analyzed with logistic regression models. Nontrivial final models (i.e., with at least one independent variable) are shown in Table 5 . The selection procedure does not leave any independent variables in models for alcohol and Stearate.

\section{Discussion}

In this prospective study, we found EtG to be associated with self-reported alcohol abuse in women with low-to-moderate alcohol consumption. However, in women, whose meconium EtG indicated a severe alcohol abuse, there was an inverse correlation with self-reported alcohol abuse, indicating that women with severe alcohol abuse might most likely be the ones to deny the alcohol abuse.

Our results might be a hint that women who drink more heavily during their pregnancy are not likely to admit their drinking status truthfully compared to those who moderately consume alcohol. 
TABLE 4: Association of socioeconomic parameters and reported alcohol abuse for Group 2 (EtG $\geq$ cutoff of $120 \mathrm{ng} / \mathrm{g}$ ). Count, percentage, and $P$ value of nonparametric test are shown.

\begin{tabular}{|c|c|c|c|c|}
\hline Patient characteristic & Total & $\begin{array}{l}\text { Alcohol consumption } \\
\text { not reported in Group } 2\end{array}$ & $\begin{array}{l}\text { Alcohol consumption } \\
\text { reported in Group } 2\end{array}$ & $P$ value \\
\hline \multicolumn{5}{|l|}{ Age } \\
\hline$<20$ & 1 & $100.0 \%$ & $0.0 \%$ & \\
\hline $20-30$ & 12 & $91.7 \%$ & $8.3 \%$ & \\
\hline $30-40$ & 38 & $68.4 \%$ & $31.6 \%$ & \\
\hline $40+$ & 3 & $33.3 \%$ & $66.7 \%$ & \\
\hline Total & 54 & & & 0.03 \\
\hline \multicolumn{5}{|l|}{ Education } \\
\hline No university-entrance diploma & 20 & $80.0 \%$ & $20.0 \%$ & \\
\hline University-entrance diploma & 34 & $67.6 \%$ & $32.4 \%$ & \\
\hline Total & 54 & & & 0.51 \\
\hline \multicolumn{5}{|l|}{ Marital status } \\
\hline Not married & 17 & $82.4 \%$ & $17.6 \%$ & \\
\hline Married & 37 & $67.6 \%$ & $32.4 \%$ & \\
\hline Total & 54 & & & 0.34 \\
\hline \multicolumn{5}{|l|}{ Income } \\
\hline$<500 €$ & 1 & $100.0 \%$ & $0.0 \%$ & \\
\hline $500-1000 €$ & 0 & $0.0 \%$ & $0.0 \%$ & \\
\hline $1000-2000 €$ & 6 & $66.7 \%$ & $33.3 \%$ & \\
\hline $2000-3000 €$ & 18 & $72.2 \%$ & $27.8 \%$ & \\
\hline $3000-4000 €$ & 5 & $80.0 \%$ & $20.0 \%$ & \\
\hline $4000-5000 €$ & 6 & $66.7 \%$ & $33.3 \%$ & \\
\hline$>5000 €$ & 3 & $66.7 \%$ & $33.3 \%$ & \\
\hline Total & 39 & & & 0.89 \\
\hline \multicolumn{5}{|l|}{ Smoking } \\
\hline No & 51 & $72.5 \%$ & $27.5 \%$ & \\
\hline Yes & 3 & $66.7 \%$ & $33.3 \%$ & \\
\hline Total & 54 & & & 0.31 \\
\hline \multicolumn{5}{|l|}{ EPDS } \\
\hline No depression & 43 & $67.4 \%$ & $32.6 \%$ & \\
\hline Slight depression & 4 & $75.0 \%$ & $25.0 \%$ & \\
\hline Moderate-to-strong depression & 3 & $100.0 \%$ & $0.0 \%$ & \\
\hline Total & 50 & & & 0.32 \\
\hline \multicolumn{5}{|l|}{ HAMD } \\
\hline No depression & 48 & $72.9 \%$ & $27.1 \%$ & \\
\hline Slight-to-moderate depression & 5 & $60.0 \%$ & $40.0 \%$ & \\
\hline Strong depression & 1 & $100.0 \%$ & $0.0 \%$ & \\
\hline Total & 54 & & & 0.79 \\
\hline
\end{tabular}

The cutoff values for objective alcohol parameters, which are currently found and tested in science and research, are not yet established due to several reasons. Nutritional and other environmental factors can influence the amount of nonoxidative alcohol metabolites [29]. There are small amounts of FAEE in meconium of neonates without active maternal alcohol consumption, which may originate from endogenous ethanol or from ethanol traces contained in common foods [30-32]. In contrast to the analysis of EtG and FAEEs in the patients' hair, there are no established and scientifically tested cutoff values for differentiating the mothers' drinking behavior via meconium, and imagining a reliable, scientifically correct, and ethical way to test the cutoff values in pregnant women is hardly possible [33]. Taking this into consideration, a strict differentiation between teetotaler, low-to-moderate, and high-risk drinkers cannot be realized. The absolute values of FAEEs and EtG in meconium can only provide a hint about the degree of the mother's ethanol 
TABLE 5: Various multiple logistic regression analyses (final models). The area under the curve (AUC) and odds ratios with 95\% confidence interval and corresponding $P$ values are shown.

\begin{tabular}{|c|c|c|c|c|c|}
\hline Target variable & AUC & Predictive variables & Odds ratio & 95\% confidence interval & $P$ value \\
\hline \multirow[t]{6}{*}{ Linoleate $(0 />0)$} & 0.58 & Education & & & \\
\hline & & No university-entrance diploma & 1 & & \\
\hline & & University-entrance diploma & 1.74 & {$[0.93,3.31]$} & 0.09 \\
\hline & & Income & & & \\
\hline & & $<500 €$ & 1 & & \\
\hline & & Per category & 0.84 & {$[0.67,1.05]$} & 0.13 \\
\hline \multirow[t]{3}{*}{ Oleate $(0 />0)$} & 0.53 & Smoking & & & \\
\hline & & No & 1 & & \\
\hline & & Yes & 2.05 & {$[0.91,4.42]$} & 0.07 \\
\hline \multirow[t]{3}{*}{ Palmitate $(0 />0)$} & 0.54 & Marital status & & & \\
\hline & & Not married & 1 & & \\
\hline & & Married & 1.57 & {$[0.86,2.79]$} & 0.13 \\
\hline \multirow[t]{9}{*}{ Myristate $(0 />0)$} & 0.58 & Age & & & \\
\hline & & $<25$ & 1 & & \\
\hline & & $\geq 25$ & 3.15 & {$[1.06,11.74]$} & 0.05 \\
\hline & & Marital status & & & \\
\hline & & Not married & 1 & & \\
\hline & & Married & 0.62 & {$[0.35,1.09]$} & 0.09 \\
\hline & & Income & & & \\
\hline & & $<500 €$ & 1 & & \\
\hline & & Per category & 0.85 & {$[0.72,1.01]$} & 0.07 \\
\hline \multirow[t]{6}{*}{ EtG $(<120 / \geq 120)$} & 0.59 & Education & & & \\
\hline & & No university-entrance diploma & 1 & & \\
\hline & & University-entrance diploma & 1.81 & {$[0.86,3.92]$} & 0.12 \\
\hline & & Income & & & \\
\hline & & $<500 €$ & 1 & & \\
\hline & & Per category & 0.79 & {$[0.60,1.04]$} & 0.10 \\
\hline
\end{tabular}

consumption. Using meconium as the material for analysis, we can overlook the last trimenon of pregnancy, as meconium is accumulated in the fetal gut from about the 20th week of gestation until birth, and the major amount of it is observed during the last weeks before birth. Therefore, at least 75\% of the sample material originates from the last 8 weeks of pregnancy $[34,35]$.

Although we had a rather large sample with 986 participants and 557 analyzed meconium samples, the positive cases-according to the toxicological meconium measureswere only a small percentage of the participants. Consecutively, the statistical power was also limited and no crossvalidation or adjustment for multiple testing was possible.

In other studies, FAEEs and EtG were already used as parameters to identify alcohol drinking mothers, and they showed partly drastic differences between the admitted drinking behavior and the one shown by using objective parameters [20-22, 36-38]. Our data are based on a large sample, and we use not only the mothers' statements, but also psychological questionnaires and objective alcohol parameters alongside each other.

Questionnaires and laboratory blood parameters used in the common routine of alcohol diagnostics are not reliable sources of information in pregnant women [2, 16, 17]. An objective evaluation of drinking status can be achieved using direct alcohol metabolites, such as EtG and FAEEs [18, 21, 28, 39], which have been shown to be parameters for the mothers' alcohol consumption [2, 19, 20, 40, 41].

Although there is no established cutoff value, Moore et al. [40] concluded that a total cumulative FAEE concentration of $>10,000 \mathrm{ng} / \mathrm{g}$ may indicate that the newborn has been exposed to significant amounts of alcohol during gestation. Three of the meconium samples in our study showed higher values, with two of those women completely denying alcohol abuse during pregnancy.

Most of the women denied alcohol consumption and most had completely negative results concerning EtG; moreover, the mean value and rank-based tests showed higher values in the group of women admitting alcohol consumption during pregnancy. These findings seem logical if we act on the assumption that the women answered the question of drinking ethanol truthfully. Still, we found the characteristic cutoff to be at $120 \mathrm{ng} / \mathrm{g}$ of EtG, under which the women's admitting status correlated positively with the meconium measures, and above which the correlation was inverse. This paradox phenomenon invigorates the findings of many other 
studies that suggest an unreliability of maternal statements concerning alcohol consumption during pregnancy $[1,2,33]$.

\section{Conclusion}

There is an ongoing immense need for further investigations in the field of alcohol consumption during pregnancy, because in Germany alone, every year, about 4000 newborn children suffer from the FAS, and even more are those born with symptoms of the fetal alcohol spectrum disease. After validation, alcohol screening could be implemented systematically in prenatal care $[42,43]$ as well as the postnatal identification of women being at high risk for child neglect $[44,45]$.

We found that women with a high alcohol consumption are more likely to deny their alcohol abuse. This finding is of clinical and scientific importance. Identifying women with a severe alcohol problem as the ones who would most likely not admit their problem might indicate an even higher risk for the unborn child, as those pregnancies are more difficult to identify. Additionally, prediction models using self-reported alcohol abuse might be more complicated as there is a positive correlation with truth in women with lowto-moderate consumption and an inverse correlation with truth in women with severe alcohol abuse. More studies are needed especially confirming meconium measurements with clinical parameters concerning fetal and pediatric outcome to test their reliability concerning clinical and scientific use.

\section{Conflict of Interests}

The authors declare that there is no conflict of interests regarding the publication of this paper.

\section{Authors' Contribution}

The FRAMES project was initiated by Tamme W. Goecke, Johannes Kornhuber, and Peter A. Fasching, and the clinical data acquisition was conducted by Tamme W. Goecke, and Matthias W. Beckmann. The meconium study was initiated by Johannes Kornhuber as an add-on project to FRAMES. Peter A. Fasching, Anne Engel, and Lothar Häberle performed the quality control of the database and statistical analysis. Tamme W. Goecke, Florian Faschingbauer, and Franziska Voigt instructed and supervised the doctoral candidates. Pascal Burger conducted the meconium processing and was the psychiatric consultant and coordinator for the project. Abdulsallam Bakdash, Michael Rothe, and Fritz Pragst performed the biochemical measurements in the meconium samples. Tamme W. Goecke, Peter A. Fasching, Pascal Burger, Johannes Kornhuber, Nicolai Maass, Lothar Häberle, and Anne Engel wrote the paper. Tamme W. Goecke and Pascal Burger have equally contributed to this work.

\section{Acknowledgments}

The authors acknowledge the support of Deutsche Forschungsgemeinschaft and Friedrich-Alexander-Universität Erlangen-Nuremberg (FAU) within the funding programme
Open Access Publishing. The authors thank the staff members of the Department of Obstetrics and the Department of Psychiatry in Erlangen, especially the doctoral candidates and assistant medical technicians involved. All authors discussed the results and commented on and approved the paper.

\section{References}

[1] C. Derauf, A. R. Katz, and D. Easa, "Agreement between maternal self-reported ethanol intake and tobacco use during pregnancy and meconium assays for fatty acid ethyl esters and cotinine," American Journal of Epidemiology, vol. 158, no. 7, pp. 705-709, 2003.

[2] F. M. Wurst, E. Kelso, W. Weinmann, F. Pragst, M. Yegles, and I. Sundström Poromaa, "Measurement of direct ethanol metabolites suggests higher rate of alcohol use among pregnant women than found with the AUDIT-a pilot study in a population-based sample of Swedish women," American Journal of Obstetrics and Gynecology, vol. 198, no. 4, pp. 407.el-407.e5, 2008.

[3] N. Raymond, C. Beer, C. Glazebrook, and K. Sayal, "Pregnant women's attitudes towards alcohol consumption," BMC Public Health, vol. 9, article 175, 2009.

[4] P. A. Fried, C. M. O’Connell, and B. Watkinson, “60- and 72month follow-up of children prenatally exposed to marijuana, cigarettes, and alcohol: cognitive and language assessment," Journal of Developmental and Behavioral Pediatrics, vol. 13, no. 6, pp. 383-391, 1992.

[5] A. C. Huizink and E. J. H. Mulder, "Maternal smoking, drinking or cannabis use during pregnancy and neurobehavioral and cognitive functioning in human offspring," Neuroscience and Biobehavioral Reviews, vol. 30, no. 1, pp. 24-41, 2006.

[6] M. Robert, A. Carceller, V. Domken et al., "Physical and neurodevelopmental evaluation of children adopted from Eastern Europe," Canadian Journal of Clinical Pharmacology, vol. 16, no. 3, pp. e432-e440, 2009.

[7] U. Kesmodel, K. Wisborg, S. F. Olsen, T. B. Henriksen, and N. J. Secher, "Moderate alcohol intake during pregnancy and the risk of stillbirth and death in the first year of life," American Journal of Epidemiology, vol. 155, no. 4, pp. 305-312, 2002.

[8] C. M. O’Leary, N. Nassar, J. J. Kurinczuk et al., "Prenatal alcohol exposure and risk of birth defects," Pediatrics, vol. 126, no. 4, pp. e843-e850, 2010.

[9] M. L. Martínez-Frías, E. Bermejo, E. Rodríguez-Pinilla, and J. L. Frías, "Risk for congenital anomalies associated with different sporadic and daily doses of alcohol consumption during pregnancy: a case-control study," Birth Defects Research A, vol. 70, no. 4, pp. 194-200, 2004.

[10] M. Todorow, T. E. Moore, and G. Koren, "Investigating the effects of low to moderate levels of prenatal alcohol exposure on child behaviour: a critical review," Journal of Population Therapeutics and Clinical Pharmacology, vol. 17, no. 2, pp. e323e330, 2010.

[11] A. M. Dunning, C. S. Healey, C. Baynes et al., "Association of ESR1 gene tagging SNPs with breast cancer risk," Human Molecular Genetics, vol. 18, no. 6, pp. 1131-1139, 2009.

[12] M. Robinson, W. H. Oddy, N. J. McLean et al., "Low-moderate prenatal alcohol exposure and risk to child behavioural development: a prospective cohort study," British Journal of Obstetrics and Gynaecology, vol. 117, no. 9, pp. 1139-1150, 2010.

[13] Y. J. Kelly, A. Sacker, R. Gray et al., "Light drinking during pregnancy: still no increased risk for socioemotional difficulties 
or cognitive deficits at 5 years of age?" Journal of Epidemiology and Community Health, vol. 66, no. 1, pp. 41-48, 2012.

[14] Y. Kelly, A. Sacker, R. Gray, J. Kelly, D. Wolke, and M. A. Quigley, "Light drinking in pregnancy, a risk for behavioural problems and cognitive deficits at 3 years of age?" International Journal of Epidemiology, vol. 38, no. 1, pp. 129-140, 2009.

[15] V. Gijsen, N. Fulga, F. Garcia-Bournissen, and G. Koren, "Does light drinking during pregnancy improve pregnancy outcome? A critical commentary," The Canadian Journal of Clinical Pharmacology, vol. 15, no. 3, pp. e782-e786, 2008.

[16] N. Kenan, A. Larsson, O. Axelsson, and A. Helander, "Changes in transferrin glycosylation during pregnancy may lead to falsepositive carbohydrate-deficient transferrin (CDT) results in testing for riskful alcohol consumption," Clinica Chimica Acta, vol. 412, no. 1-2, pp. 129-133, 2011.

[17] J. D. Cook, "Biochemical markers of alcohol use in pregnant women," Clinical Biochemistry, vol. 36, no. 1, pp. 9-19, 2003.

[18] N. de Giovanni, G. Donadio, and M. Chiarotti, “The reliability of Fatty Acid Ethyl Esters (FAEE) as biological markers for the diagnosis of alcohol abuse," Journal of Analytical Toxicology, vol. 31, no. 2, pp. 93-97, 2007.

[19] D. L. Caprara, J. Klein, and G. Koren, "Diagnosis of fetal alcohol spectrum disorder (FASD): fatty acid ethyl esters and neonatal hair analysis," Annali dell'Istituto Superiore di Sanita, vol. 42, no. 1, pp. 39-45, 2006.

[20] A. Bakdash, P. Burger, T. W. Goecke et al., "Quantification of fatty acid ethyl esters (FAEE) and ethyl glucuronide (EtG) in meconium from newborns for detection of alcohol abuse in a maternal health evaluation study," Analytical and Bioanalytical Chemistry, vol. 396, no. 7, pp. 2469-2477, 2010.

[21] F. Pragst, M. Rothe, B. Moench, M. Hastedt, S. Herre, and D. Simmert, "Combined use of fatty acid ethyl esters and ethyl glucuronide in hair for diagnosis of alcohol abuse: interpretation and advantages," Forensic Science International, vol. 196, no. 13, pp. 101-110, 2010.

[22] L. Morini, E. Marchei, F. Vagnarelli et al., "Ethyl glucuronide and ethyl sulfate in meconium and hair-potential biomarkers of intrauterine exposure to ethanol," Forensic Science International, vol. 196, no. 1-3, pp. 74-77, 2010.

[23] J. R. Hutson, K. Aleksa, F. Pragst, and G. Koren, "Detection and quantification of fatty acid ethyl esters in meconium by headspace-solid-phase microextraction and gas chromatography-mass spectrometry," Journal of Chromatography B, vol. 877, no. 1-2, pp. 8-12, 2009.

[24] U. Reulbach, S. Bleich, J. Knörr et al., "Pre-, peri- and postpartal depression first cognition from FRAMES (Franconian Maternal Health Evaluation Studies)," Fortschritte der Neurologie Psychiatrie, vol. 77, no. 12, pp. 708-713, 2009.

[25] D. Mehta, C. Quast, P. A. Fasching et al., "The 5-HTTLPR polymorphism modulates the influence on environmental stressors on peripartum depression symptoms," Journal of Affective Disorders, vol. 136, no. 3, pp. 1192-1197, 2012.

[26] P. A. Fasching, F. Faschingbauer, T. W. Goecke et al., "Genetic variants in the tryptophan hydroxylase 2 gene (TPH2) and depression during and after pregnancy," Journal of Psychiatric Research, vol. 46, no. 9, pp. 1109-1117, 2012.

[27] A. Hein, C. Rauh, A. Engel et al., "Socioeconomic status and depression during and after pregnancy in the Franconian Maternal Health Evaluation Studies (FRAMES)," Archives of Gynecology and Obstetrics, 2013.

[28] F. Pragst, V. Auwaerter, F. Sporkert, and K. Spiegel, "Analysis of fatty acid ethyl esters in hair as possible markers of chronically elevated alcohol consumption by headspace solidphase microextraction (HS-SPME) and gas chromatographymass spectrometry (GC-MS)," Forensic Science International, vol. 121, no. 1-2, pp. 76-88, 2001.

[29] V. Kulaga, Y. Velazquez-Armenta, K. Aleksa, Z. Vergee, and G. Koren, "Assessment and detection: the effect of hair pigment on the incorporation of fatty acid ethyl esters (FAEE)," Alcohol and Alcoholism, vol. 44, no. 3, pp. 287-292, 2009.

[30] A. Thierauf, H. Gnann, A. Wohlfarth et al., "Urine tested positive for ethyl glucuronide and ethyl sulphate after the consumption of "non-alcoholic" beer," Forensic Science International, vol. 202, no. 1-3, pp. 82-85, 2010.

[31] A. Thierauf, C. C. Halter, S. Rana et al., "Urine tested positive for ethyl glucuronide after trace amounts of ethanol," Addiction, vol. 104, no. 12, pp. 2007-2012, 2009.

[32] A. Thierauf, A. Wohlfarth, V. Auwärter, M. G. Perdekamp, F. M. Wurst, and W. Weinmann, "Urine tested positive for ethyl glucuronide and ethyl sulfate after the consumption of yeast and sugar," Forensic Science International, vol. 202, no. 1-3, pp. e45e47, 2010.

[33] A. E. Gifford, K. J. Farkas, L. W. Jackson et al., "Assessment of benefits of a universal screen for maternal alcohol use during pregnancy," Birth Defects Research A, vol. 88, no. 10, pp. 838846, 2010.

[34] D. Chan, J. Klein, T. Karaskov, and G. Koren, "Fetal exposure to alcohol as evidenced by fatty acid ethyl esters in meconium in the absence of maternal drinking history in pregnancy," Therapeutic Drug Monitoring, vol. 26, no. 5, pp. 474-481, 2004.

[35] L. Burd and R. Hofer, "Biomarkers for detection of prenatal alcohol exposure: a critical review of fatty acid ethyl esters in meconium," Birth Defects Research A, vol. 82, no. 7, pp. 487-493, 2008.

[36] F. Pragst and M. Yegles, "Determination of fatty acid ethyl esters (FAEE) and ethyl glucuronide (EtG) in hair: a promising way for retrospective detection of alcohol abuse during pregnancy?" Therapeutic Drug Monitoring, vol. 30, no. 2, pp. 255-263, 2008.

[37] S. Pichini, L. Morini, E. Marchei et al., "Ethylglucuronide and ethylsulfate in meconium to assess gestational ethanol exposure: preliminary results in two Mediterranean cohorts," The Canadian Journal of Clinical Pharmacology, vol. 16, no. 2, pp. e370-e375, 2009.

[38] L. Morini, A. Groppi, E. Marchei et al., "Population baseline of meconium ethyl glucuronide and ethyl sulfate concentrations in newborns of nondrinking women in 2 Mediterranean cohorts," Therapeutic Drug Monitoring, vol. 32, no. 3, pp. 359-363, 2010.

[39] S. Süße, C. M. Selavka, T. Mieczkowski, and F. Pragst, "Fatty acid ethyl ester concentrations in hair and self-reported alcohol consumption in 644 cases from different origin," Forensic Science International, vol. 196, no. 1-3, pp. 111-117, 2010.

[40] C. Moore, J. Jones, D. Lewis, and K. Buchi, "Prevalence of fatty acid ethyl esters in meconium specimens," Clinical Chemistry, vol. 49, no. 1, pp. 133-136, 2003.

[41] Y. I. Goh, J. R. Hutson, L. Lum et al., "Rates of fetal alcohol exposure among newborns in a high-risk obstetric unit," Alcohol, vol. 44, no. 7-8, pp. 629-634, 2010.

[42] B. Yazdi, H. Abele, E. M. Grischke et al., "Obstetrics. Prenatal care in reconstruction: from head to toe?" Geburtsh Frauenheilk, vol. 73, no. 4, pp. 295-298, 2013.

[43] P. Bühring, "Fetales Alkoholsyndrom: zu hundert Prozent vermeidbar,” Deutsches Ärzteblatt, vol. 105, no. 43, pp. A-2258/B1931/C-1879, 2008. 
[44] T. Besier, M. Pillhofer, S. Botzenhart et al., "Child abuse and neglect: screening for risks during the perinatal period," Geburtsh Frauenheilk, vol. 72, no. 5, pp. 397-402, 2012.

[45] W. Schwab, C. Marth, and A. M. Bergant, "Post-traumatic stress disorder post partum: the impact of birth on the prevalence of post-traumatic stress disorder (PTSD) in multiparous women," Geburtshilfe und Frauenheilkunde, vol. 72, no. 1, pp. 56-63, 2012. 


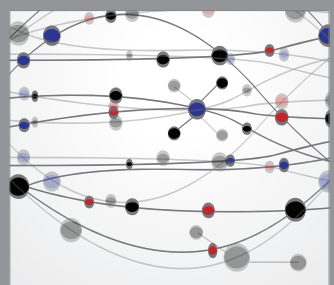

The Scientific World Journal
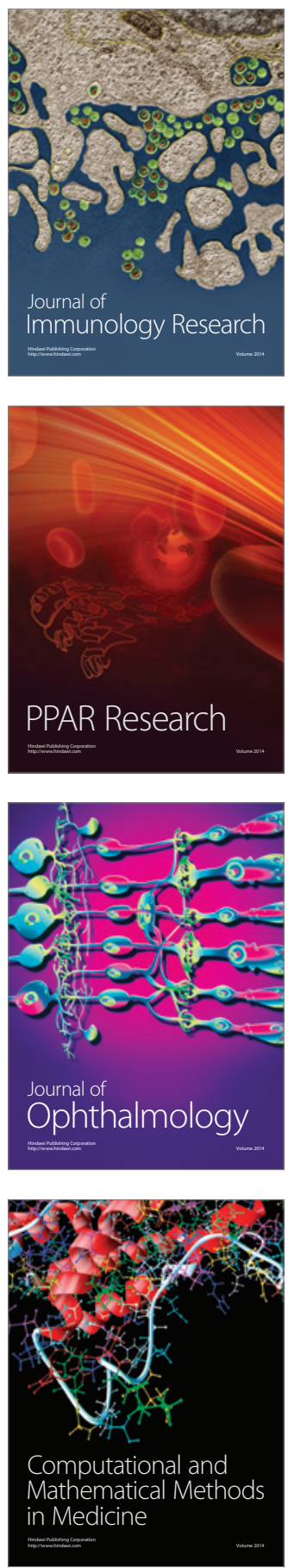

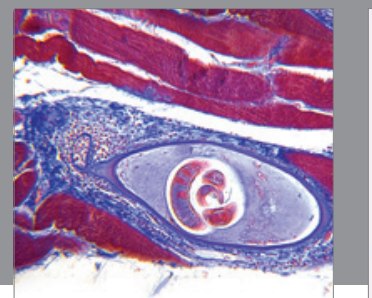

Gastroenterology

Research and Practice
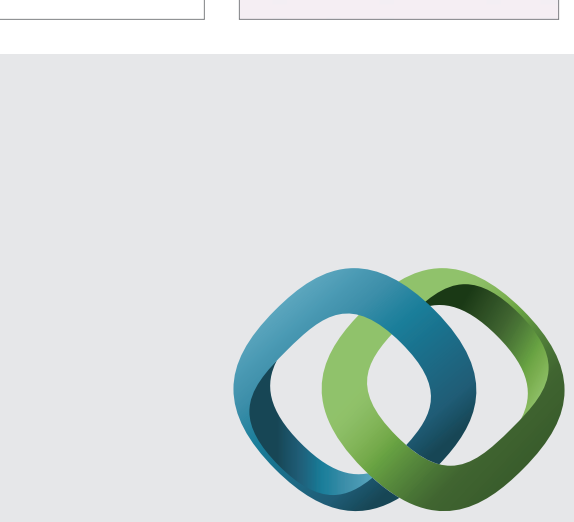

\section{Hindawi}

Submit your manuscripts at

http://www.hindawi.com
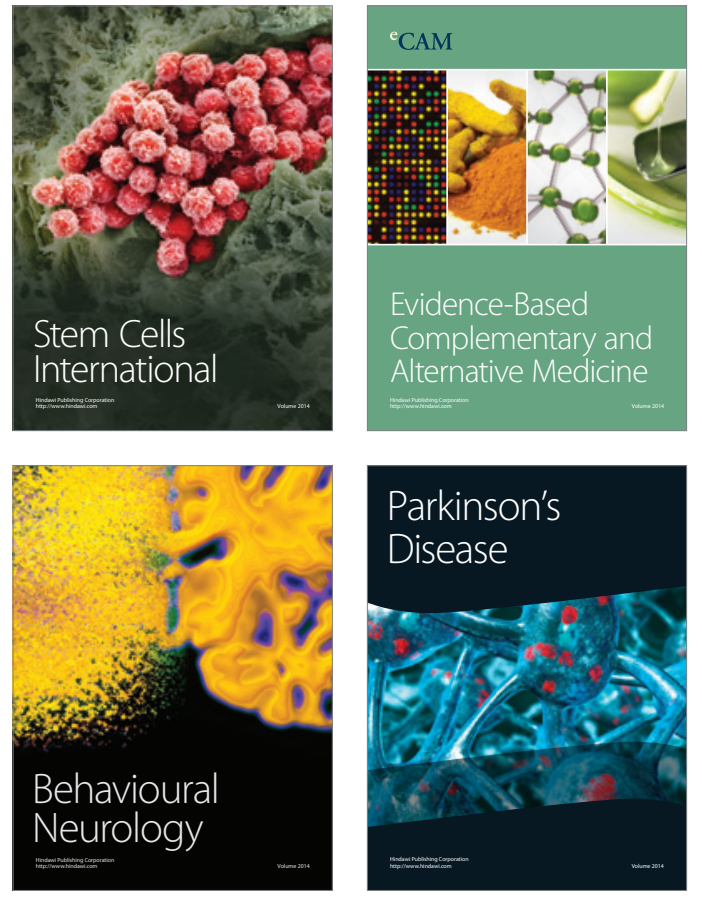
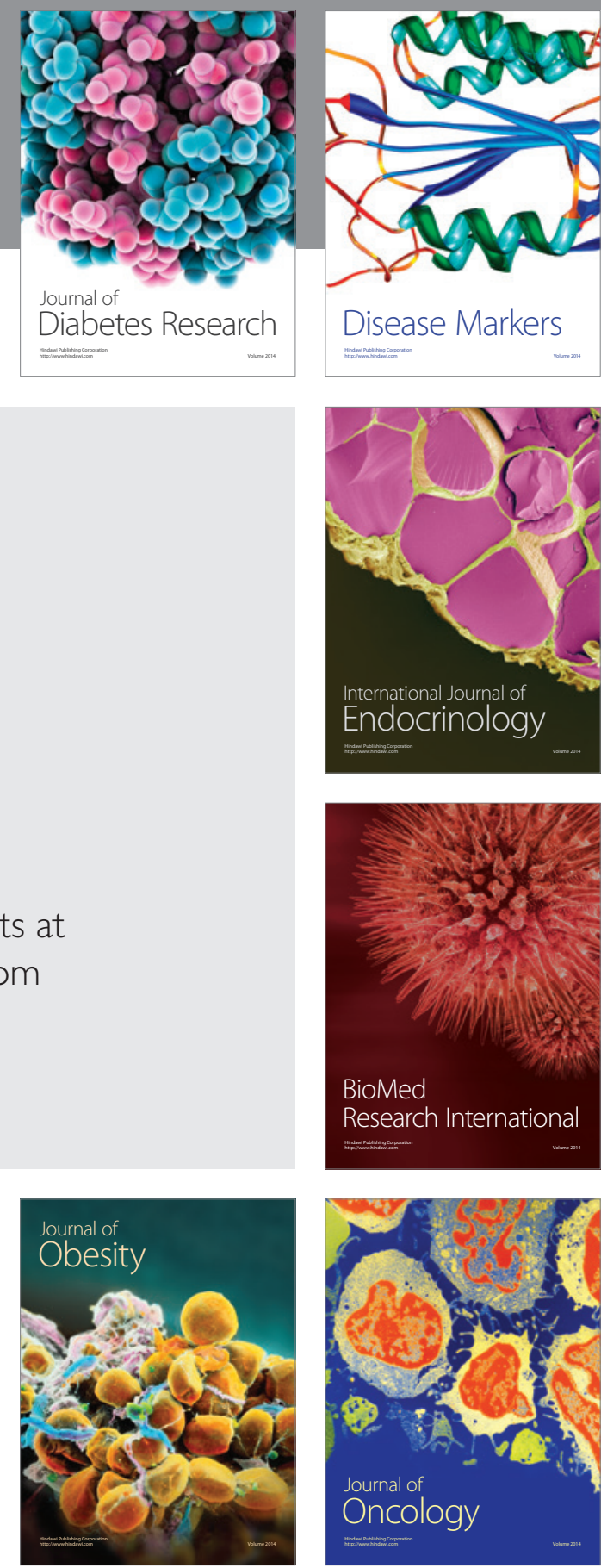

Disease Markers
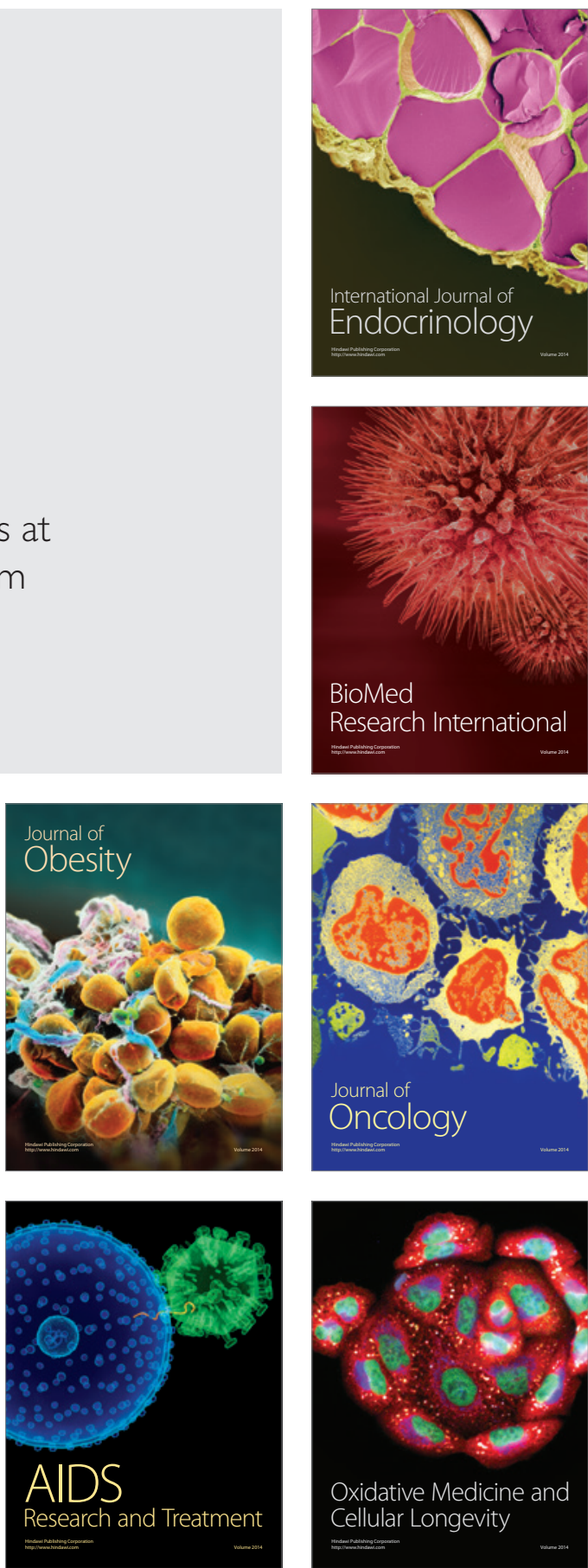